\title{
A POLYNOMIAL-TIME ALGORITHM FOR THE TOPOLOGICAL TYPE OF A REAL ALGEBRAIC CURVE -EXTENDED ABSTRACT
}

\author{
DENNIS S. ARNON AND SCOTT MCCALLUM
}

\section{Dedicated to the memory of Gus Efroymson}

1. Introduction. Let $f(x, y, z)$ be a homogeneous polynomial with rational coefficients. Let $C_{f}$ be the real projective curve defined by $f=0$. It is well known [9] that if $C_{f}$ is nonsingular, then it is a compact one-dimensional manifold, and so homeomorphic to a disjoint union of circles. A circle can have either a one-sided or two-sided imbedding in $\mathbf{R} P^{2}$; in the latter case it has both an interior (homeomorphic to a disk), and an exterior (homeomorphic to a Möbius strip). The two-sided components of $C_{f}$ are called ovals. If $f$ has even degree, then every component of $C_{f}$ is an oval; if degree $(f)$ is odd, every component except one is an oval.

Curves $C_{1}$ and $C_{2}$ have the same topological type if there is a homeomorphism $\varphi: \mathbf{R} P^{2} \rightarrow \mathbf{R} P^{2}$ which maps $C_{1}$ onto $C_{2}$. Each oval of a nonsingular curve $C_{f}$ is either inside or outside any other; the partial ordering of the ovals induced by this inclusion relation, together with the parity of the degree of $f$, determine the topological type of the curve.

We present an algorithm which, given $f(x, y, z)$ with rational coefficients, determines whether $C_{f}$ is nonsingular, and if so, determines the ordering of its ovals.

2. Description of algorithm. We may assume that $f$ is squarefree; (if not, we can replace $f$ by its greatest squarefree divisor $h$, as $C_{f}=C_{k}$ ). The main step of the algorithm is construction of a cellular decomposition $D_{f}$ of $\mathbf{R} P^{2}$ such that every component of $C_{f}$ is a union of cells of $D_{f}$. The following description of $D_{f}$ is produced: (1) a list of the pairs of adjacent cells (two cells are adjacent if their union is connected), and (2) a list of the cells contained in $C_{f}$. In the course of constructing $D_{f}$ we determine if $C_{f}$ has singularities, and if so, halt.

The first author would like to acknowledge the support of the Purdue University Research Foundation.

The second author would like to acknowledge the support of the National Science Foundation, Grant MCS-8009357. 
Assuming $C_{f}$ is nonsingular, the rest of the algorithm is straightforward. The reflexive transitive closure $\bar{R}$ of the adjacency relation is an equivalence relation; for a subset $X$ of $\mathbf{R} P^{2}$, let $\bar{R}(X)$ denote the restriction of $\bar{R}$ to the cells of $D_{f}$ which meet $X$. We construct the equivalence classes of $\bar{R}\left(C_{f}\right)$; (the union of) each class is a component of $C_{f}$. Let $O$ be one of these components and $K$ the corresponding class of $\bar{R}\left(C_{f}\right)$. We construct the equivalence classes of $\bar{R}$ (complement $(O)$ ); (the union of) each class is a component of complement $(O) . O$ is an oval if and only if there are two such classes; if there is only one, we do not process $O$ further. Suppose there are two classes $K_{1}$ and $K_{2}$. Let $V$ be the union of the cells of $K_{1}$ and let $W$ be the union of the cells of $K_{2}$. We want to determine which of $V$ and $W$ is the interior $(\operatorname{Int}(O))$ and which is the exterior $(\operatorname{Ext}(O))$ of $O$. Now it can be shown that $D_{f}$ gives $\mathbf{R} P^{2}$ the structure of a finite cell complex. As $O \cup V=\bar{V}$ and $O \cup W=\bar{W}, K \cup K_{1}$ and $K \cup K_{2}$ give $\bar{V}$ and $\bar{W}$ respectively the structure of subcomplexes of $\mathbf{R} P^{2}$. We can therefore compute the Euler characteristic $\chi$ of each of $\bar{V}$ and $\bar{W}$ using the formula

$$
\chi=\alpha_{0}-\alpha_{1}+\alpha_{2},
$$

where $\alpha_{i}$ is the number of $i$-cells. But $\overline{\operatorname{Int}(O)}$ is homeomorphic to the closed disc and $\overline{\operatorname{Ext}(O)}$ is homeomorphic to the closed Möbius band. Thus $\chi \overline{(\operatorname{Int}(O)})=1$ and $\chi \overline{(\operatorname{Ext}(O)})=0$. Hence we can determine from $\chi$ which of $\bar{V}$ and $\bar{W}$ is $\overline{\operatorname{Int}(O)}$ and which is $\overline{\operatorname{Ext}(O)}$. After making this determination for all ovals of $C_{f}$, we know, for any oval, which cells of $D_{f}$ are inside, which on, and which outside it. From this information the ordering of ovals follows.

The chief tool for constructing $D_{f}$ is the cylindrical algebraic decomposition (cad) algorithm [2], [3], [4]. We use this algorithm to construct a cellular decomposition of the affine plane relative to the polynomial $g(x, y)=f(x, y, 1)$. (The algorithm would compute the discriminant $D(x)$ of $g(x, y)$, isolate the real roots of $D(x)$, and then "lift" the cells in the real line which are determined by the roots of $D(x)$ to cells in the plane which are determined by the locus of $g(x, y)$. Note that $D(x)$ is not the zero polynomial as $f(x, y, z)$, and hence $g(x, y)$, is squarefree.) Regarding the affine plane as a subset of $\mathbf{R} P^{2}$, we extend to a cellular decomposition of $\mathbf{R} P^{2}$ relative to $f(x, y, z)$ by appropriately partitioning the line at infinity into cells. Before applying the cad algorithm, we perform a linear change of coordinates of $\mathbf{R} P^{2}$, if necessary, to ensure that the curve $C_{f}$ has only simple intersections with the line at infinity, and that $C_{f}$ does not contain the point $[0,1,0]$. These properties facilitate the passage from the affine to the projective plane.

3. Concluding remarks. It can be shown that the computing time of our 
algorithm is $O(p(n, d))$, for some polynomial function $p$ of the degree $n$ of $f$ and the size $d$ of its coefficients. Polotovskii [7] gave a topological type algorithm for curves of even degree, but did not establish a bound for it. His approach is quite different from ours; he examines the curves $f(x, y, z)$ $+\varepsilon z^{n},(n=\operatorname{degree}(f))$, for various small values of $\varepsilon$. As noted by Fuks and Delzell [5], one could get a topological type algorithm from Tarski's decision procedure for elementary algebra and geometry [8], but such an algorithm would have an exponential computing time bound. We have recently learned of an independently developed topological type algorithm by Gianni and Traverso [6], which has some resemblance to our method, but does not make use of cell complexes.

Because the time of our method depends almost entirely on the time required by the cad algorithm, and because the cad algorithm has recently been implemented [1], our algorithm appears to have some practical value. It could be used, for example, to study examples relating to Hilbert's 16th problem [9].

4. Acknowledgements. We are indebted to G. Brumfiel for the observation that Euler characteristic suffices to distinguish the interior of an oval from its exterior. We had originally envisioned a full homology calculation. The second author would like to acknowledge helpful and inspiring conversations on the subject of this paper with the following people: G. Collins, E. Fadell, T.-C. Kuo, E. Mansfield.

\section{REFERENCES}

1. D. S. Arnon, Algorithms for the geometry of semi-algebraic sets, Technical Report \#436, Computer Science Dept., University of Wisconsin, Madison, Wisconsin, (Ph. D. thesis), 1981.

2. - G. E. Collins and S. McCallum, Cylindrical algebraic decomposition I: the basic algorithm. SIAM J. Comp. 13 (1984).

3. - and Cylindrical algebraic decomposition $\mathrm{II}$ : an adjacency algorithm for the plane. SIAM J. Comp. 13 (1984).

4. G. E. Collins, Quantifier elimination for real closed fields by cylindrical algebraic decomposition, in Proceedings of the Second GI Conference on Automata and Formal Languages, Lecture notes in Computer Science 33 Springer-Verlag, Berlin, 1975, 134163.

5. C. Delzell, private communication, 1980.

6. P. Gianni and C. Traverso: Shape determination for real curves and surfaces, Publication 23, Dipartimento di Matematica, Università di Pisa, July, 1983.

7. G. M. Polotovskii, Algorithm for determining the topological type of a rough plane algebraic curve of even degree, Gorkov Gos. Univ. Učen. Zap, Vyp 187 (1973), 143187.

8. A. Tarski, A Decision Method for Elementary Algebra and Geometry, University of California Press, 1951.

9. G. Wilson, Hilbert's Sixteenth Problem, Topology 17 (1978), 53-73. 
Xeror PARC 3333 Coyote Hill Road, Palo Alto, CA 94304

Dept. of COMPuter Science, University of Toronto, Toronto, CANADA M5S 1A7 